\title{
The stromal microenvironment provides an escape route from FLT3 inhibitors through the GAS6-AXL-STAT5 axis
}

\author{
Anna Orlova ${ }^{1}$, Heidi A. Neubauer ${ }^{1}$ and Richard Moriggl ${ }^{1,2}$ \\ ${ }^{1}$ Institute of Animal Breeding and Genetics, University of Veterinary Medicine and ${ }^{2}$ Medical University Vienna, Vienna, Austria \\ E-mail: RICHARD MORIGGL - richard.moriggl@vetmeduni.ac.at
}

doi:10.3324/haematol.2019.225862

T The FLT3-ITD mutation is one of the most common rearrangements in acute myeloid leukemia (AML), and is particularly associated with poor prognosis and recurrent development of resistance. In 2017, the FLT3 tyrosine kinase inhibitor (TKI) midostaurin was approved for use in combination with standard cytarabine-based chemotherapy. Several other small molecule inhibitors against FLT3 tyrosine kinase are currently being tested in phase III clinical trials (e.g. gilteritinib and quizartinib). Despite successful application of the targeted therapy in patients, emergence of resistance is still a major drawback in clinical practice. ${ }^{1} \mathrm{~A}$ better understanding of resistance mechanisms in cancer is key to defining better treatment strategies for patients. The new study by Dumas et al. in this issue of the Journal unravels mechanisms involving the tyrosine kinase receptor AXL contributing to the development of resistance to quizartinib in FLT3-ITD ${ }^{+}$AML. $^{2}$

AXL belongs to the family of TAM receptors, and together with two other members, TYRO3 and MER, it was first shown to have malignant roles in solid cancers. ${ }^{3,4}$ AXL was identified as one of the most prominently activated tyrosine kinase receptors in colorectal, esophageal, thyroid, breast, prostate and lung carcinomas, and its activation was associated with transforming growth factor beta (TGF $\beta$ ) signaling. ${ }^{4,5} \mathrm{AXI}$ is selectively activated by GAS6 ligand, which has a significantly higher affinity to AXL compared to the other family members. Further ligands for TAM receptors include Protein S, Tubby, Tubby-like protein 1, and Galectin-3. ${ }^{4}$ Soluble forms of AXL (sAXL) are also reported, and result from cleavage by ADAM10/17 proteases in the plasma of patients with advanced liver cancer; they are, therefore, of extremely important diagnostic value for liver cancer progression. ${ }^{6}$ TAM receptors are involved in processes promoting cell growth and survival, cell adhesion, migration, blood coagulation, and cytokine release. However, TAM receptors were also reported to impede cancer cells through stimulation of tumor cell-targeting immune cells. ${ }^{8}$

The new findings reported by Dumas et al. ${ }^{2}$ confirm an important cancer-protective role for the stromal microenvironment, mechanistically identifying that it induces cytokine production and hypoxic conditions to trigger the activation of AXL and the transcription factor STAT5 in FLT3-ITD ${ }^{+}$AML (Figure 1A). The authors further show that stroma-induced expression of $A X L$, mediated by STAT5, drives progression of the disease. The paper provides evidence that growth arrest specific protein 6 (GAS6) ligand secreted from stromal cells activates AXL and, together with hypoxia, contributes to AML progression and resistance to quizartinib. Notably, a bypass mechanism was described involving activation of the AXL receptor kinase to compensate for FLT3 inhibition to promote AML progression.

Interestingly, similar findings also implicated AXL activation, together with another receptor tyrosine kinase MET, in driving resistance mechanisms in HER2-positive gastric cancer with
TKI treatment. ${ }^{9}$ Here, the authors generated and exploited afatinib-resistant gastric cancer cell lines to identify AXL and MET as key players in the development of drug resistance. Yoshioka et al. proposed combinatorial treatment using afatinib with pan-kinase inhibitor cabozantinib, which also targets AXL/MET, to prevent development of therapy resistance or to potentially sensitize patients who have already developed resistance. ${ }^{9}$

STAT5A/B proteins are key downstream transcription factors in FLT3-ITD ${ }^{+}$AML, and they mediate signals from hyperactive FLT3. STAT5 inhibition was reported to be a promising strategy for FLT3-ITD ${ }^{+}$AML treatment. ${ }^{10-13}$ The oncogenic roles of highly tyrosine-phosphorylated STAT5 (pYSTAT5) in hematopoietic diseases were best exemplified using graded expression and activity levels of STAT5A/B in gain-of-function transgenic mouse models. ${ }^{14,15}$ Important downstream transcriptional changes triggered by STAT5 in neoplastic myeloid cells can involve enhanced expression of DNMT3A, BCL2 or D-type cyclin family members, as well as MYC induction. This panel of downstream STAT5 target genes has now been expanded to include AXL in quizartinib-resistant FLT3-ITD ${ }^{+}$AML, and it will be of particular interest to explore whether this finding is also applicable in other cancers. ${ }^{2}$ Interestingly, TET or DNMT3 genes are often mutated in AML, and both have been reported to either form protein interactions with STAT5 or undergo direct gene regulation by STAT5. In particular, mutations in chromatin modifiers TET2, DNMT3A, ASXL1, IDH1/2, as well as STAT5, were found to be of important prognostic value in FLT3-ITD ${ }^{+}$mutated AML cases. It will be important to explore further the impact of these proteins and the chromatin landscape on the GAS6-AXL-STAT5 AML progression axis. ${ }^{16,17}$

A previous study also examined the effects of PI3K/AKT/mTOR inhibitors on a FLT3-ITD ${ }^{+}$AML cell line compared with a cell line harboring point mutations within the TKD2 domain of FLT3 (FLT3-TKD). The authors reported that FLT3-ITD ${ }^{+}$cells are more resistant to the aforementioned FLT3 inhibitors compared with FLT3-TKD ${ }^{+}$cells. The authors proposed hyperactivation of STAT5 in FLT3-ITD ${ }^{+}$AML cells as a protective mechanism against PISK/AKT/mTOR inhibition. ${ }^{18}$ Interestingly, Dumas et al. showed in their model that inhibition of PIBK/AKT signaling had no effect on AXL or STAT5 phosphorylation, and, therefore, this did not directly mediate the development of resistance to therapy.'

Based on these recent findings, we used public gene expression datasets available from the Oncomine database to independently evaluate $A X L$ gene expression data from patients with AML as well as from patients with other hematopoietic cancers. ${ }^{19}$ As also discussed by Dumas et al., we found AXL to be significantly up-regulated in AML patient samples (Figure 1B). Interestingly, upregulation of $A X L$ was also clearly evident in various subtypes of B-cell and T-cell leukemias/lymphomas (Figure 1B), suggesting a potentially broader relevance for the oncogenic action of AXL upregulation in hematopoietic can- 


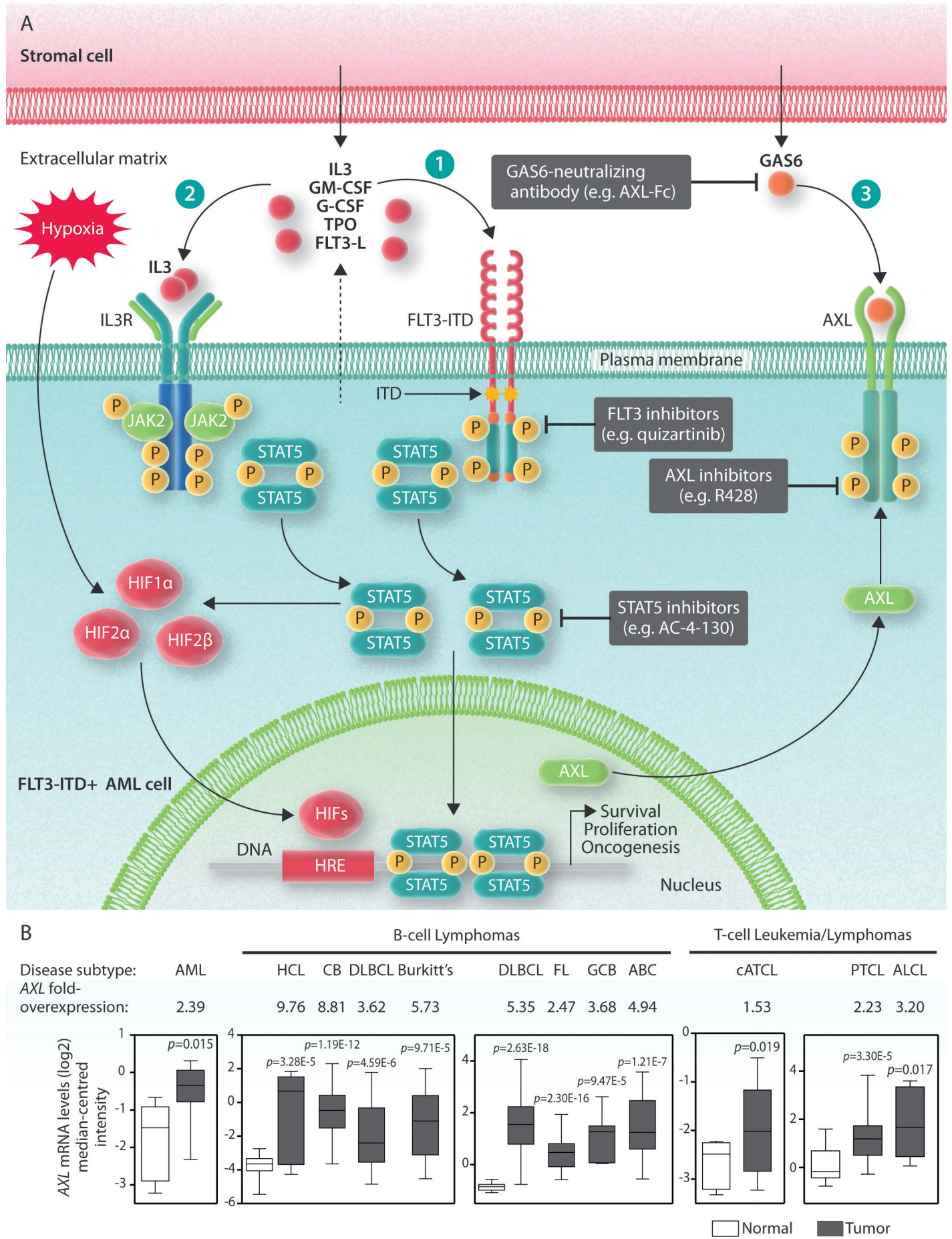

Figure 1. AXL promotes acute myeloid leukemia (AML) progression upon development of resistance. (A) Stromal cells were shown to express cytokines and ligands (see 1 and 2) (such as IL3 or FLT3-L, solid arrow) to a greater extent than AML cells (dashed arrow). Additionally, stromal cells were shown to produce GAS6 (see 3), which activates AXL receptor signaling. JAK2 binds to the cytosolic juxta-membrane region of dimeric cytokine receptors such as IL3R via the BOX1 and BOX2 receptor motifs upon stimulation by respective cytokines (see 2). JAK2 activation triggers STAT5 signaling, which promotes oncogenic gene transcription to propagate cancer cell survival, proliferation, and metabolic reprogramming. Additionally, STAT5 was shown to bind to the AXL promoter to induce expression. Mutated FLT3 (FLT3-ITD, yellow stars), frequently found in AML patients, can induce phosphorylation of STAT5 directly. Therapeutic agents used by Dumas et al. ${ }^{2}$ to target these key proteins/pathways in AML are summarized in gray boxes. Hypoxia induced by the stromal microenvironment induces expression of HIF transcription factors, and STAT5 can induce expression of HIF2 $\alpha$. Subsequently, HIF can bind to HRE elements in the AXL promoter. AML: acute myeloid leukemia; IL3: Interleukin 3; IL3R: Interleukin 3 receptor; GM-CSF: granulocyte-macrophage colony-stimulating factor; TPO: thrombopoietin; FLT3: Fms-like tyrosine kinase 3; ITD: internal tandem duplication; FLT3-L: Fms-like tyrosine kinase 3 ligand; GAS6: growth arrest-specific 6; HIF: hypoxia-inducible factor; HRE: hypoxia-response element; JAK: Janus kinase; STAT: signal transducer and activator of transcription. (B) AXL mRNA expression levels in patients with hematopoietic cancers using data from the Oncomine database. Box plots showing human hematopoietic cancers with significant upregulation of $A X L$ mRNA levels in tumor cells, compared with tissue-matched normal control cells. Data were extracted from the Oncomine database from the following studies (graphs from left to right): Stegmaier Leukemia, Basso Lymphoma, Compagno Lymphoma, Choi Leukemia, and Piccaluga Lymphoma. Data from multiple normal-tissue subtypes included in some datasets were pooled for clarity. For all analyses, the $P$-value threshold was set to 0.05 , the fold-change threshold was set to 1.5 , and the gene rank threshold was set to 'all'. HCL: hairy cell leukemia; CB: centroblastic lymphoma; DLBCL: diffuse large B-cell lymphoma; FL: follicular lymphoma; GCB: germinal center B-cell-like DLBCL; ABC: activated B-cell-like DLBCL; CATCL: chronic adult T-cell leukemia/lymphoma; PTCL: unspecified peripheral T-cell lymphoma; ALCL: anaplastic large cell lymphoma. 
cers. Notably, mining the Oncomine database additionally revealed significant overexpression of both TYRO3 and MER in various B- and T-cell leukemia/lymphoma datasets, but not in AML (data not shown). This might suggest a potentially different mechanism of oncogenic signaling in lymphoid neoplasms involving all three receptor family members, as opposed to the clear role of AXL in FLT3-ITD ${ }^{+}$ $\mathrm{AML}$; this could be a focus point for further investigations.

The new study by Dumas et al. described a resistance mechanism against the FLT3 inhibitor quizartinib, where both the FLT3-ITD mutation and the GAS6-AXL axis trigger important kinase signaling cascades. Their results suggest that a combination of FLT3- and AXL-specific inhibitors, or the exploitation of dual FLT3-AXL inhibitors (e.g. cabozantinib), might be beneficial in FLT3-ITD ${ }^{+}$AML patients at risk of relapse. Furthermore, selective STAT5 inhibitors have the potential to become an effective tool for targeted and combinatorial therapy in AML.

In summary, FLT3 inhibitors display efficacy in the treatment of FLT3-ITD-driven AML, but more efficient targeting of AML blasts remains an unresolved medical need to fight therapeutic resistance, and to improve the poor overall survival and quality of life of patients.

\section{Funding}

HAN and RM are supported by the Austrian Science Fund (FWF) [SFB-F04707, SFB-F06105, and under the frame of ERA PerMed (I 4218-B) and ERA-NET (I 4157-B)]. RM, HAN and $A O$ were also generously supported by a private donation from Liechtenstein.

\section{References}

1. Daver N, Schlenk RF, Russell NH, et al. Targeting FLT3 mutations in AML: review of current knowledge and evidence. Leukemia. 2019;33(2):299-312.

2. Dumas PY, Naudin C, Maetin-Lannerée S, et al. Hematopoietic niche drives FLT3-ITD acute myeloid leukemia resistance to quizartinib via STAT5- and hypoxia- dependent up-regulation of AXL.
Haematologica. 2019;104(10):2017-2027.

3. Kirane A, Ludwig KF, Sorrelle N, et al. Warfarin Blocks Gas6-Mediated Axl Activation Required for Pancreatic Cancer Epithelial Plasticity and Metastasis. Cancer Res. 2015;75(18):3699-3705

4. Wu X, Liu X, Koul S, et al. AXL kinase as a novel target for cancer therapy. Oncotarget. 2014:5(20):9546-9563.

5. Lee HJ, Jeng YM, Chen YL, et al. Gas6/Axl pathway promotes tumor invasion through the transcriptional activation of Slug in hepatocellular carcinoma. Carcinogenesis. 2014;35(4):769-775.

6. Holstein E, Binder M, Mikulits W, et al. Dynamics of Axl Receptor Shedding in Hepatocellular Carcinoma and Its Implication for Theranostics. Int J Mol Sci. 2018;19(12).

7. Linger RMA, Keating AK, Earp HS, et al. TAM Receptor Tyrosine Kinases: Biologic Functions, Signaling, and Potential Therapeutic Targeting in Human Cancer. Adv Cancer Res. 2008;100:35-83

8. Paolino M, Penninger JM. The Role of TAM Family Receptors in Immune Cell Function: Implications for Cancer Therapy. Cancers (Basel). 2016;8(10).

9. Yoshioka T, Shien K, Takeda T, et al. Acquired resistance mechanisms to afatinib in HER2-amplified gastric cancer cells. Cancer Sci. 2019 Jun 4. [Epub ahead of print]

10. Mizuki M, Schwable J, Steur C, et al. Suppression of myeloid transcription factors and induction of STAT response genes by AML-specific Flt3 mutations. Blood. 2003;101(8)3164-3173.

11. Nelson EA, Walker SR, Xiang M, et al. The STAT5 Inhibitor Pimozide Displays Efficacy in Models of Acute Myelogenous Leukemia Driven by FLT3 Mutations. Genes Cancer. 2012;3(7-8):503-511.

12. Wingelhofer B, Maurer B, Heyes EC, et al. Pharmacologic inhibition of STAT5 in acute myeloid leukemia. Leukemia. 2018;32(5):1135-1146.

13. Yoshimoto G, Miyamoto T, Jabbarzadeh-Tabrizi S, et al. FLT3-ITD upregulates MCL-1 to promote survival of stem cells in acute myeloid leukemia via FLT3-ITD-specific STAT5 activation. Blood. 2009:114(24):5034-5043.

14. Maurer B, Nivarthi $H$, Wingelhofer B, et al. High activation of STAT5A drives peripheral T-cell lymphoma and leukemia. Haematologica. 2019 May 23. [Epub ahead of print]

15. Pham HTT, Maurer B, Prchal-Murphy M, et al. STAT5BN642H is a driver mutation for $\mathrm{T}$ cell neoplasia. J Clin Invest. 2018;128(1):387-401.

16. Bowman RL, Busque L, Levine RL, et al. Clonal Hematopoiesis and Evolution to Hematopoietic Malignancies. Cell Stem Cell. 2018;22(2):157-170

17. Fuster IJ and Walsh K. Somatic Mutations and Clonal Hematopoiesis. Circ Res. 2018;122(3):523-532.

18. Nogami A, Oshikawa G, Okada K, et al. FLT3-ITD confers resistance to the PI3K/Akt pathway inhibitors by protecting the mTOR/4EBP1/Mcl-1 pathway through STAT5 activation in acute myeloid leukemia. Oncotarget. 2016;6(11):9189-9205.

19. Rhodes DR, Yu J, Shanker K, et al. ONCOMINE: a cancer microarray database and integrated data-mining platform. Neoplasia. 2004;6(1):1-6.

\title{
Pediatric aplastic anemia treatment patterns and responses; power in the numbers
}

\author{
Lucy C. Fox ${ }^{1,2}$ and David S. Ritchie ${ }^{2,3}$
}

${ }^{1}$ Diagnostic Haematology, Peter MacCallum Cancer Centre; ${ }^{2}$ University of Melbourne and ${ }^{3}$ Clinical Haematology, Peter MacCallum Cancer Centre and Royal Melbourne Hospital, Melbourne, Australia

E-mail: DAVID S. RITCHIE - david.ritchie@mh.org.au

doi:10.3324/haematol.2019.225870

I n this edition of Haematologica, Rogers et al., representing 25 individual institutions, collectively report on their findings of the diagnostic approaches, applied therapies and responses in a cohort of 314 pediatric patients (aged 1-20 years) with a diagnosis of aplastic anemia (AA) collected through the North American Pediatric Aplastic Anemia Consortium (NAPAAC). ${ }^{1}$ This study highlights a number of important messages; specifically the power of collated registry data in a rare disease, the need to retest and continually refine diagnostic crite- ria to be fit for real-world purposes, the patterns of response and relapse to immunosuppressive therapy (IST) in a pediatric setting including the substantial differences in outcomes from IST in children compared with that in adults, and the importance of allogeneic stem cell transplant (HCT) in the therapy of refractory or relapsed disease.

Although AA can affect people at any stage of life, with a well reported bimodal peak of age incidence observed in older children/young adults and those over sixty years, 